\title{
Robot-assisted Landing of VTOL UAVs: Design and Comparison of Coupled and Decoupling Linear State-space Control Approaches
}

\author{
Moritz Maier ${ }^{1}$, André Oeschger, and Konstantin Kondak ${ }^{1}$
}

\begin{abstract}
This paper addresses the problem of landing a VTOL UAV using a serial robotic manipulator fixed to the landing surface, which assists the UAV during the last, most challenging, landing phase. In this phase, UAV and manipulator are connected via a universal hinge, which decouples the flying vehicle's and the robot's end-effector orientation. This novel system is meant to be used for VTOL UAVs landing on moving platforms under severe environmental conditions. The main contribution of the paper is the design of a linear state-space controller for position and orientation of the UAV while it is fixed to the manipulator. Furthermore, we compare a coupled and a decoupling realization of the model-based controller with a model-free controller. Both model-based controllers consider the dynamics of an attitude-controlled aerial vehicle and use the acceleration of the robot's end-effector as control input. The decoupling controller allows to fully actuate the UAV using the manipulator in addition to the UAV's actuators. All three controllers are validated and compared in experiments using a KUKA/DLR light-weight robot on a non-moving base and an AR.Drone 2.0 quadrotor. The experimental results show that decoupling is superior to the coupled and the model-free approach, since the orientation of the $U A V$ rotorcraft is controlled more precisely.
\end{abstract}

Index Terms-Aerial Robotics, Direct/Inverse Dynamics Formulation, Motion Control of Manipulators

\section{INTRODUCTION}

$\mathbf{U}$ NMANNED aerial vehicles with vertical take-off and landing capabilities (VTOL UAVs) are nowadays widely used, e.g. for aerial photography and filming, surveillance or inspection tasks [1]. Recent enhancements of the aerial platforms and advances in autonomous functionalities have increased their operational range and the deployment of aerial vehicles under severe environmental conditions has become technically attainable. Recovering the UAV at the end of the mission is a crucial task, for instance for marine applications, such as search of castaways, ice monitoring, or inspection of offshore wind farms, due to heavy wind gusts and ship motions [2]. It is apparent that in these applications a special procedure is needed in order to safely land UAV rotorcrafts on moving platforms and that this procedure has to be robust against external disturbances like wind gusts. One approach

Manuscript received: August, 31, 2015; Revised November, 14, 2015; Accepted October, 28, 2015.

This paper was recommended for publication by Editor Jonathan Roberts upon evaluation of the Associate Editor and Reviewers' comments.

${ }^{1}$ Moritz Maier and Konstantin Kondak are with the German Aerospace Center (DLR), Institute of Robotics and Mechatronics, Münchener Straße 20, 82234 Weßling, Germany moritz.maierdalr.de

Digital Object Identifier (not yet available)

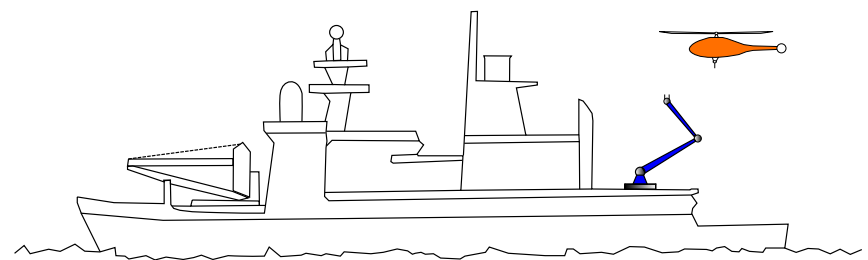

Fig. 1. Schematic sketch of a novel landing system for VTOL UAVs with a robotic manipulator (blue) and a UAV rotorcraft (orange).

to this problem is to use special sensor equipment [3] and to incoporate wind observance and platform motion in the controller design of the UAV [4], [5], [6], [7], but this will fail as soon as the actuators of the aerial vehicle reach their limits. Another possible solution are assistance systems, such as hexapods [8], tethers [9], [10] or mobile platforms [11].

We present an alternative solution in [12] with an increased workspace compared to available approaches and incorporate an autonomous clamping mechanism to fix the UAV to the assistance system. Our system employs a robot manipulator, as depicted in Fig. 1, and should be able to capture an unmanned aerial vehicle in-flight. The advantages of the robotic landing system compared to the aforementioned approaches are that it

- does not require additional power of the UAV's engine,

- can also be used for take-off, while the possible take-off weight is increased if a forward velocity is induced,

- and is able to compensate the platform motion within the boundaries of the robot's dexterous workspace.

The landing system can be used on different platforms and is therefore suitable for many scenarios where physical robotrobot-interaction is involved. For example in a heterogeneous team consisting of a ground based vehicle equipped with the manipulator and a flying robot, which is captured and landed for recharging.

This paper focuses on the final landing phase in which the aerial vehicle is fixed to the robot manipulator. The position and orientation of the UAV should be controlled by the manipulator such that the aerial vehicle is not tilted in an undesired direction. This is comparable to the conventional inverted pendulum problem with the difference that the UAV already has an onboard attitude controller. In [12], we tackled this problem by designing a nonlinear backstepping controller, which takes advantage of the cascaded structure of the system and for which Lyapunov stability was shown. Controlling both position and orientation simultaneously using the manipulator 
was not possible, because the input matrix for the underactuated system is not invertible. Decoupling and full actuation of the position and orientation subsystems respectively was achieved by controlling the position of the pivot point of the universal hinge and the orientation of the VTOL UAV about its center of mass while sending attitude commands to the UAV.

Therefore, in this paper, we design a linear state-space controller with the acceleration of the end-effector as control input and show that this approach relaxes the assumptions made in [12]. It allows to design coupled and decoupling controllers for orientation and position of the attitude-controlled VTOL UAV. Coupled means that orientation and position of the aerial vehicle are both controlled by the robot manipulator. On the contrary, decoupling means that the position of the UAV is controlled by the robot such that changes of the UAV's attitude do not affect its center of mass position. The latter is again achieved by sending attitude commands to the UAV and has the effect that the complete system becomes fully actuated.

This work is structured as follows: In Section II, the equations of motion of a rotary-wing UAV fixed at the end-effector of a robotic manipulator via a universal hinge are introduced. In addition, a stability criterion for linear proportionalderivative UAV attitude controllers is derived. A feed-forward torque control law for the serial robot is presented in Section III allowing to control a desired end-effector acceleration. The linearized dynamic model from Section II is then utilized for the design of three different realizations of a state-space controller. In Section IV, the results of experiments with the VTOL UAV landing system demonstrator on a non-moving base and an off-the-shelf quadrotor are presented and the controllers are compared and evaluated. Finally, the work is concluded and an outlook is given in Section V.

\section{VTOL UAV AND MANIPUlator System Modeling}

In this section, the equations of motion of an unmanned aerial vehicle fixed to a robot manipulator by means of a universal hinge are presented (Subsection II-A), linearized, and analyzed for stability (Subsection II-B).

\section{A. Equations of Motion of a UAV Rotorcraft}

The rotational dynamics in the body-fixed frame $b$ and the translational dynamics in the inertial frame $i$ of a small-scale rotary-wing UAV (Fig. 2) can concisely be written as [12]

$$
\begin{aligned}
{\left[\begin{array}{cc}
\boldsymbol{J} & \mathbf{0}_{3 \times 3} \\
\mathbf{0}_{3 \times 3} & m \boldsymbol{I}_{3 \times 3}
\end{array}\right] } & \left(\begin{array}{c}
{ }^{b} \dot{\boldsymbol{\omega}} \\
{ }^{i} \boldsymbol{\boldsymbol { v }}
\end{array}\right)_{\mathrm{UAV}}= \\
& \left(\begin{array}{c}
-\boldsymbol{S}\left({ }^{b} \boldsymbol{\omega}\right) \boldsymbol{J}^{b} \boldsymbol{\omega}+{ }^{b} \boldsymbol{\tau} \\
-m g \boldsymbol{e}_{3}+{ }^{i} \boldsymbol{F}
\end{array}\right)_{\mathrm{UAV}},
\end{aligned}
$$

where $m$ is the mass of the $\mathrm{UAV}, \boldsymbol{J}=\operatorname{diag}\left(J_{x x}, J_{y y}, J_{z z}\right)$ is its diagonal inertia tensor with respect to its center of mass $C M$, ${ }^{b} \boldsymbol{\omega}_{\text {UAV }}$ is its rotational, and ${ }^{i} \boldsymbol{v}_{\text {UAV }}$ its translational velocity. $\boldsymbol{S}(\cdot)$ denotes a skew-symmetric matrix, i.e.

$$
\boldsymbol{S}(\boldsymbol{n})=\left[\begin{array}{ccc}
0 & -n_{3} & n_{2} \\
n_{3} & 0 & -n_{1} \\
-n_{2} & n_{1} & 0
\end{array}\right] .
$$

In (1), $g$ is the gravitational acceleration and $\boldsymbol{e}_{i}$ denotes a unit vector, e.g. $\boldsymbol{e}_{3}=\left(\begin{array}{lll}0 & 0 & 1\end{array}\right)^{T}$. ${ }^{b} \boldsymbol{\tau}_{\mathrm{UAV}}$ comprises torques

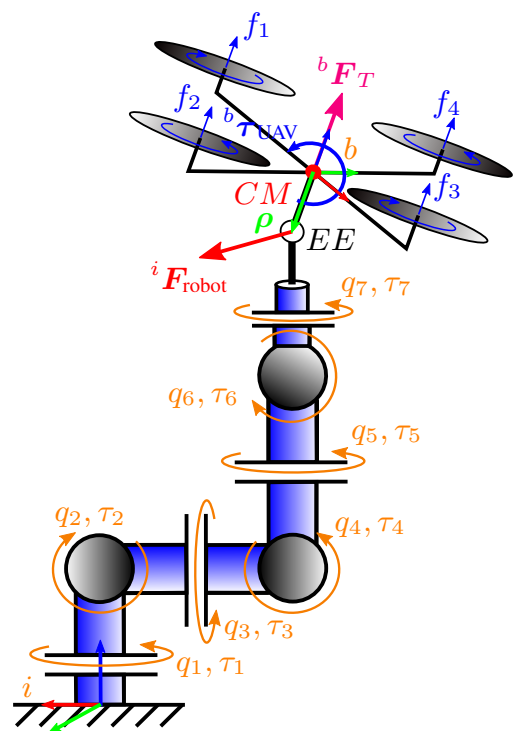

Fig. 2. Model of UAV quadrotor connected to a serial robotic manipulator with seven degrees-of-freedom.

about the UAV's center of mass $C M$ and ${ }^{i} \boldsymbol{F}_{\text {UAV }}$ is the sum of the forces acting on the UAV in addition to gravity. From a torque balance for the UAV connected to the manipulator via a universal hinge, we obtain

$$
{ }^{b} \boldsymbol{\tau}_{\mathrm{UAV}}={ }^{b} \boldsymbol{\tau}_{a t t}+\boldsymbol{S}\left({ }^{b} \boldsymbol{\rho}\right) \boldsymbol{R}_{b i}(\varphi, \theta, \psi){ }^{i} \boldsymbol{F}_{\text {robot }}
$$

Therein, $\boldsymbol{R}_{b i}(\varphi, \theta, \psi)$ is a rotation matrix constructed using Euler angles $\boldsymbol{\Phi}=\left(\begin{array}{lll}\varphi & \theta & \psi\end{array}\right)^{T}$, that transfers a vector given in the inertial frame $i$ to the body-fixed frame $b$. The force applied by the robot at its end-effector $E E$ is denoted as ${ }^{i} \boldsymbol{F}_{\text {robot }}{ }^{b} \boldsymbol{\rho}$ is the displacement vector written in $b$ from $C M$ to $E E$ defined as ${ }^{b} \boldsymbol{\rho}=\left(\begin{array}{lll}0 & 0 & -l\end{array}\right)^{T}$, with the length $l$ of the universal hinge. The torque needed to stabilize a desired attitude is denoted as ${ }^{b} \boldsymbol{\tau}_{\text {att }}$ in (3). VTOL UAVs like helicopters and quadrotors are generally underactuated with the thrust vector ${ }^{b} \boldsymbol{F}_{T}=\left(\begin{array}{lll}0 & 0 & f_{T}\end{array}\right)^{T}$ assumed to be always perpendicular to the $\left({ }^{b} x,{ }^{b} y\right)$-plane [13]. For a quadrotor as shown in Fig. 2 the collective thrust $f_{T}$ is the sum of all rotor thrust forces $\sum_{i=1}^{4} f_{i}$. When the UAV is connected to the robot manipulator, a balance of forces yields

$$
{ }^{i} \boldsymbol{F}_{\mathrm{UAV}}=f_{T} \boldsymbol{R}_{b i}^{T}(\varphi, \theta, \psi) \boldsymbol{e}_{3}+{ }^{i} \boldsymbol{F}_{\text {robot }} .
$$

Combining equations (1), (3), and (4) with the kinematics

$$
\begin{aligned}
{ }^{i} \dot{\boldsymbol{r}}_{C M} & ={ }^{i} \boldsymbol{v}_{\mathrm{UAV}}, \\
\dot{\boldsymbol{R}}_{b i}^{T} & =\boldsymbol{R}_{b i}^{T} \boldsymbol{S}\left({ }^{b} \boldsymbol{\omega}_{\mathrm{UAV}}\right),
\end{aligned}
$$

gives the complete equations of motion of the VTOL UAV fixed to the robot manipulator via a universal hinge. Therein ${ }^{i} \boldsymbol{r}_{C M}$ is the position of the aerial vehicle's center of mass in the inertial frame. Note that in order to make the equations tractable for controller design, we assume that the UAV does not affect the motion of the manipulator.

\section{B. Stability Criterion for Linear PD Attitude Controllers}

The question arises whether it is possible for a given attitude controller to stabilize the orientation while the UAV is rigidly 
connected to the end-effector of the manipulator. Therefore, we proceed with analyzing the stability of the hover state $\left(\begin{array}{ll}{ }^{b} \boldsymbol{\omega}^{T} & \boldsymbol{\Phi}^{T}\end{array}\right)^{T}=\mathbf{0}_{6 \times 1}$ with constant thrust force $f_{T}=m g$. In static equilibrium the sum of all forces is zero. Therefore, it follows from (1) and (4) that ${ }^{i} \boldsymbol{F}_{\mathrm{UAV}}=m g \boldsymbol{e}_{3}$.

It is reasonable to assume that the VTOL UAV is equipped with a linear proportional-derivative (PD) attitude controller [13] and that the UAV's rotors produce the torque

$$
\begin{aligned}
{ }^{b} \boldsymbol{\tau}_{a t t}= & -\operatorname{diag}\left(k_{\omega_{x}}, k_{\omega_{y}}, k_{\omega_{z}}\right){ }^{b} \omega_{\mathrm{UAV}} \\
& -\operatorname{diag}\left(k_{\varphi}, k_{\theta}, k_{\psi}\right)\left(\begin{array}{c}
\varphi \\
\theta \\
\psi
\end{array}\right) .
\end{aligned}
$$

In the following, $x$ - and $y$-direction are combined for conciseness. This is indicated using the notation $x / y$ which means that the equation can be written out using the elements before or after the slash, respectively. Inserting (3), (4), and (7) in (1) and linearizing about the hover state yields for the rotational dynamics about the $x-, y$-, and $z$-axis respectively (see Fig. 2)

$$
\begin{aligned}
\left(\begin{array}{c}
{ }^{\dot{\omega}_{x / y}} \\
\dot{\varphi} / \dot{\theta}
\end{array}\right) & =\underbrace{\left[\begin{array}{cc}
\frac{-k_{\omega_{x / y}}}{J_{x x / y y}} & \frac{-k_{\varphi / \theta}+m g l}{J_{x x / y y}} \\
1 & 0
\end{array}\right]}_{\boldsymbol{A}_{x / y}}\left(\begin{array}{c}
{ }^{\omega_{x / y}} \\
\varphi / \theta
\end{array}\right), \\
\left(\begin{array}{c}
b_{\dot{\omega}_{z}} \\
\dot{\psi}
\end{array}\right) & =\underbrace{\left[\begin{array}{cc}
\frac{-k_{\omega_{z}}}{J_{z z}} & \frac{-k_{\psi}}{J_{z z}} \\
1 & 0
\end{array}\right]}_{\boldsymbol{A}_{z}}\left(\begin{array}{c}
b \omega_{z} \\
\psi
\end{array}\right) .
\end{aligned}
$$

The characteristic polynomials $P_{i}=\operatorname{det}\left(\boldsymbol{A}_{i}-\lambda_{i} \boldsymbol{I}_{2 \times 2}\right)$ for $i \in\{x, y, z\}$ are obtained as

$$
\begin{aligned}
P_{x / y} & =\lambda_{x / y}^{2}+\frac{k_{\omega_{x / y}}}{J_{x x / y y}} \lambda_{x / y}+\frac{k_{\varphi / \theta}-m g l}{J_{x x / y y}}, \\
P_{z} & =\lambda_{z}^{2}+\frac{k_{\omega_{z}}}{J_{z z}} \lambda_{z}+\frac{k_{\psi}}{J_{z z}} .
\end{aligned}
$$

According to the Routh-Hurwitz criterion [14], the hover state is stable if all coefficients within (10) and (11) are positive. Since all other parameters are positive, it follows that the gains $k_{\varphi / \theta}, k_{\omega_{x / y}}, k_{\psi}$, and $k_{\omega_{z}}$ have to satisfy

$$
k_{\varphi / \theta}>m \cdot g \cdot l, \quad k_{\omega_{x / y}}>0, \quad k_{\psi}>0, \quad k_{\omega_{z}}>0 .
$$

It can be concluded that the aerial vehicle can be connected to the manipulator via a universal hinge without considering the UAV's orientation in the controller for the landing system if the UAV's linear PD attitude controller fullfills criterion (12). Moreover, from the eigenfrequency $\omega_{0}$ and the damping $\zeta$ of (10)

$$
\begin{aligned}
& \omega_{0}=\sqrt{\frac{k_{\varphi / \theta}-m g l}{J_{x x / y y}},} \\
& \zeta=\frac{k_{\omega_{x / y}}}{2 \sqrt{J_{x x / y y}\left(k_{\varphi / \theta}-m g l\right)}},
\end{aligned}
$$

it can be seen that by increasing the length $l$ of the universal hinge or the mass $m$ of the UAV, the eigenfrequency $\omega_{0}$ can be decreased while the damping $\zeta$ is increased and vice versa.

\section{Controller Design}

In this section, a state-space control approach for the robotic landing system for VTOL UAVs is presented. We use the

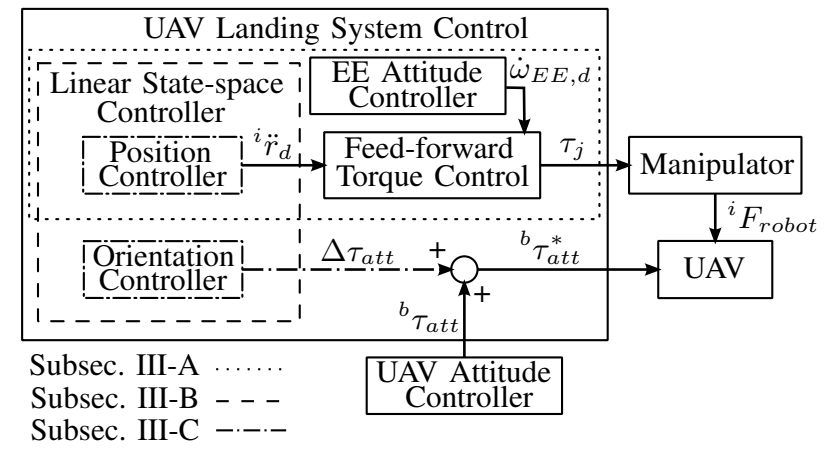

Fig. 3. Scheme of the state-space control approach with the three realizations of the controller depicted using different line styles. The controller (19) for the orientation of the end-effector as well as the feed-forward torque controller (21) from Subsection III-A are used for all three realizations. The coupled controller (26) - (28) in Subsection III-B produces a desired translational acceleration of the robot's end-effector to control both position and orientation of the VTOL UAV simultaneously. In order to fulfill the same task, the decoupling controller (34)-(35) in Subsection III-C generates an appropriate end-effector acceleration and a torque which is sent to the attitude controller of the aerial vehicle.

linearized model introduced in Section II of the UAV fixed to the manipulator's end-effector $E E$ with the acceleration of the end-effector as control input. The overall controller scheme with its three different realizations is shown in Fig. 3. In Subsection III-A, the feed-forward control law for a torquecontrolled robot is derived allowing to command a desired endeffector acceleration. A primer linear state-space controller is designed, neglecting the dynamics of the VTOL UAV. This controller is then extended in Subsection III-B in order to control both the UAV's center of mass position $C M$ and its orientation about $C M$ in a coupled manner using the robot manipulator. The attitude controller of the UAV is assumed to be linear with known gains, as presented in Subsection II-B. Finally, we use a modal control approach to decouple the acceleration of the end-effector from the orientation of the VTOL UAV in Subsection III-C.

\section{A. Position and Orientation Control of a Manipulator}

The dynamics in joint coordinates $\boldsymbol{q} \in \Theta^{n}$ of a serial robotic manipulator with $n$ joints are given as

$$
\boldsymbol{M}(\boldsymbol{q}) \ddot{\boldsymbol{q}}+\boldsymbol{C}(\boldsymbol{q}, \dot{\boldsymbol{q}}) \dot{\boldsymbol{q}}+\boldsymbol{g}(\boldsymbol{q})=\boldsymbol{\tau}_{j},
$$

wherein $\boldsymbol{q}_{n \times 1}, \boldsymbol{M}_{n \times n}, \boldsymbol{C}_{n \times n}$, and $\boldsymbol{g}_{n \times 1}$ are the vector of joint positions, the mass matrix, the Coriolis/centrifugal matrix, and the vector of gravity terms respectively [15]. We assume that we use a robot, e.g. the DLR/KUKA light-weight robot [15], where the joint torques $\boldsymbol{\tau}_{j}$ can be controlled directly. The joint angles $\boldsymbol{q}$ and the cartesian end-effector position and orientation $(\boldsymbol{r}, \phi)_{E E} \in \mathbb{R}^{6}$ are related by the forward kinematics of the robot $\boldsymbol{f}: \Theta^{n} \rightarrow \mathbb{R}^{6}$. The relationship between forces at the end-effector $\boldsymbol{F}_{E E} \in \mathbb{R}^{6}$ and joint torques are given for a manipulator with $n>6$ by

$$
\boldsymbol{\tau}_{j}=\underbrace{\mathcal{J}(\boldsymbol{q})^{T} \boldsymbol{F}_{E E}}_{\boldsymbol{\tau}_{c t r l}}+\underbrace{\left(\boldsymbol{I}-\left(\mathcal{J}(\boldsymbol{q})^{+} \mathcal{J}(\boldsymbol{q})\right)^{T}\right) \boldsymbol{\Gamma}_{0}}_{\boldsymbol{\tau}_{n s p}},
$$

where $\mathcal{J}(\boldsymbol{q})^{T}=\frac{\partial \boldsymbol{f}(\boldsymbol{q})}{\partial \boldsymbol{q}}$ is the Jacobian, $\mathcal{J}(\boldsymbol{q})^{+}$is a generalized inverse of the Jacobian, and $\Gamma_{0} \in \mathbb{R}^{6}$ defines generalized 
forces in the nullspace of the robot [15]. In (16), $\boldsymbol{\tau}_{\text {ctrl }}$ is the part of $\boldsymbol{\tau}_{j}$ which is used to control the motion of the end-effector, whereas $\boldsymbol{\tau}_{n s p}$ are nullspace torques, i.e. torques that do not affect the end-effector motion but the posture of the robot manipulator. In order to mitigate the influence of the robot's motor inertias, of friction in the joints, and of model uncertainties, the integral of the position error $\tilde{\boldsymbol{r}}=\boldsymbol{r}_{E E, d}-\boldsymbol{r}_{E E}$ is added to the system states. We define the translational acceleration of the end-effector $\ddot{\boldsymbol{r}}_{E E, d}:=\ddot{\boldsymbol{r}}_{E E}$ as an intermediate control input. Assuming direct control of the acceleration, we define a linear state-space model for the translational motion of the end-effector

$$
\left(\begin{array}{c}
\ddot{\boldsymbol{r}}_{E E} \\
\dot{\boldsymbol{r}}_{E E} \\
\tilde{\boldsymbol{r}}
\end{array}\right)=\left[\begin{array}{ccc}
0 & 0 & 0 \\
\boldsymbol{I} & 0 & 0 \\
0 & -\boldsymbol{I} & 0
\end{array}\right]\left(\begin{array}{c}
\dot{\boldsymbol{r}}_{E E} \\
\boldsymbol{r}_{E E} \\
\int \tilde{\boldsymbol{r}} \mathrm{dt}
\end{array}\right)+\left(\begin{array}{c}
\ddot{\boldsymbol{r}}_{E E, d} \\
0 \\
\boldsymbol{r}_{E E, d}
\end{array}\right)
$$

and design a linear controller

$$
\ddot{\boldsymbol{r}}_{E E, d}=-\boldsymbol{K}_{1}\left(\dot{\boldsymbol{r}}_{E E} \quad \boldsymbol{r}_{E E} \quad \int \tilde{\boldsymbol{r}} \mathrm{dt}\right)^{T}
$$

such that the poles of the closed-loop are placed at a desired location in the left complex half-plane using e.g. Ackermann's formula [14]. Since UAV and manipulator are connected via the rotational hinge, the orientation of the aerial vehicle and the robotic end-effector can be controlled independently. We adapt a control law from [16] in order to generate a desired rotational acceleration for the robot

$$
\begin{aligned}
\dot{\omega}_{E E, d}= & -\frac{1}{2} k_{E E}\left(\begin{array}{l}
\left(\boldsymbol{R}_{E E} \boldsymbol{R}_{d}\right)^{\{3,2\}}-\left(\boldsymbol{R}_{E E} \boldsymbol{R}_{d}\right)^{\{2,3\}} \\
\left(\boldsymbol{R}_{E E} \boldsymbol{R}_{d}\right)^{\{1,3\}}-\left(\boldsymbol{R}_{E E} \boldsymbol{R}_{d}\right)^{\{3,1\}} \\
\left(\boldsymbol{R}_{E E} \boldsymbol{R}_{d}\right)^{\{2,1\}}-\left(\boldsymbol{R}_{E E} \boldsymbol{R}_{d}\right)^{\{1,2\}}
\end{array}\right) \\
& -k_{\omega_{E E}} \boldsymbol{\omega}_{E E}
\end{aligned}
$$

using the actual and the desired orientation $\boldsymbol{R}_{E E}$ and $\boldsymbol{R}_{d}$ as well as the rotational part of the velocity of the end-effector

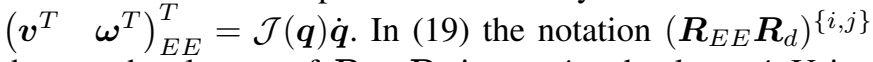
denotes the element of $\boldsymbol{R}_{E E} \boldsymbol{R}_{d}$ in row $i$ and column $j$. Using (15), the cartesian acceleration of the end-effector can be obtained from

$$
\begin{aligned}
\left(\begin{array}{c}
\ddot{\boldsymbol{r}} \\
\dot{\boldsymbol{\omega}}
\end{array}\right)_{E E} & =\frac{\mathrm{d}}{\mathrm{d} t}(\mathcal{J}(\boldsymbol{q}) \dot{\boldsymbol{q}})=\dot{\mathcal{J}}(\boldsymbol{q}) \dot{\boldsymbol{q}}+\mathcal{J}(\boldsymbol{q}) \ddot{\boldsymbol{q}} \\
& =\dot{\mathcal{J}}(\boldsymbol{q}) \dot{\boldsymbol{q}}+\mathcal{J}(\boldsymbol{q}) \boldsymbol{M}(\boldsymbol{q})^{-1}\left(\boldsymbol{\tau}_{j}-\boldsymbol{C}(\boldsymbol{q}, \dot{\boldsymbol{q}}) \dot{\boldsymbol{q}}-\boldsymbol{g}(\boldsymbol{q})\right) .
\end{aligned}
$$

We neglect the term $\dot{\mathcal{J}}(\boldsymbol{q}) \dot{\boldsymbol{q}}$ in (20), since its numeric value is small compared to the other terms, and find the final feedforward joint torque control law for the manipulator which yields $\left(\ddot{\boldsymbol{r}}^{T} \quad \dot{\boldsymbol{\omega}}^{T}\right)_{E E}^{T}=\left(\begin{array}{ll}\ddot{\boldsymbol{r}}^{T} & \dot{\boldsymbol{\omega}}^{T}\end{array}\right)_{E E, d}^{T}$ to be

$$
\boldsymbol{\tau}_{j}=\boldsymbol{M}(\boldsymbol{q}) \mathcal{J}(\boldsymbol{q})^{+}\left(\begin{array}{c}
\ddot{\boldsymbol{r}} \\
\dot{\boldsymbol{\omega}}
\end{array}\right)_{E E, d}+\boldsymbol{C}(\boldsymbol{q}, \dot{\boldsymbol{q}}) \dot{\boldsymbol{q}}+\boldsymbol{g}(\boldsymbol{q})+\boldsymbol{\tau}_{n s p} .
$$

Alternatively, the rigid-body dynamics of the manipulator in cartesian space can be utilized, as done in [12], in order to achieve tracking of a desired cartesian velocity. The evaluation of this alternative approach is intentionally left for future work. In the next section, the controller (17) is extendend in order to stabilize the position and the orientation of the UAV simultaneously.

\section{B. Coupled Position and Orientation Control of a UAV}

As soon as the aerial vehicle is connected to the manipulator via the universal hinge, the position of its center of mass in the inertial frame is given by

$$
{ }^{i} \boldsymbol{r}_{C M}={ }^{i} \boldsymbol{r}_{E E}+\boldsymbol{R}_{i b}{ }^{b} \boldsymbol{\rho} .
$$

We assume that the UAV is equipped with the linear attitude controller (7) and the thrust force is $f_{T}=m g$. Therefore, we obtain the following independent linear state-space models for the $x-, y$-, and $z$-motion of the UAV

$$
\begin{aligned}
& \left(\begin{array}{c}
\ddot{r}_{x} \\
\dot{r}_{x} \\
\dot{\omega}_{y} \\
\dot{\theta}
\end{array}\right)_{U A V}=\underbrace{\left[\begin{array}{cccc}
0 & 0 & 0 & g \\
1 & 0 & 0 & 0 \\
0 & 0 & & \boldsymbol{A}_{y} \\
0 & 0 &
\end{array}\right]}_{\overline{\boldsymbol{A}}_{x}}\left(\begin{array}{c}
\dot{r}_{x} \\
r_{x} \\
\omega_{y} \\
\theta
\end{array}\right)_{U A V}+\left(\begin{array}{c}
1 \\
0 \\
-\frac{m l}{J_{y y}} \\
0
\end{array}\right) \ddot{r}_{E E, x}, \\
& \left(\begin{array}{c}
\ddot{r}_{y} \\
\dot{r}_{y} \\
\dot{\omega}_{x} \\
\dot{\varphi}
\end{array}\right)_{U A V}=\underbrace{\left[\begin{array}{cccc}
0 & 0 & 0 & -g \\
1 & 0 & 0 & 0 \\
0 & 0 & \boldsymbol{A}_{x}
\end{array}\right]}_{\overline{\boldsymbol{A}}_{y}}\left(\begin{array}{c}
\dot{r}_{y} \\
r_{y} \\
\omega_{x} \\
\varphi
\end{array}\right)_{U A V}+\left(\begin{array}{c}
1 \\
0 \\
\frac{m l}{J_{x x}} \\
0
\end{array}\right) \ddot{r}_{E E, y}, \\
& \left(\begin{array}{c}
\ddot{r}_{z} \\
\dot{r}_{z} \\
\dot{\omega}_{z} \\
\dot{\psi}
\end{array}\right)_{U A V}=\left[\begin{array}{cccc}
0 & 0 & 0 & 0 \\
1 & 0 & 0 & 0 \\
0 & 0 & \boldsymbol{A}_{z}
\end{array}\right]\left(\begin{array}{c}
\dot{r}_{z} \\
r_{z} \\
\omega_{z} \\
\psi
\end{array}\right)_{U A V}+\left(\begin{array}{l}
1 \\
0 \\
0 \\
0
\end{array}\right) \ddot{r}_{E E, z} .
\end{aligned}
$$

It can be seen from (25) that the yaw angle $\psi$ of the UAV is not controllable by $\ddot{\boldsymbol{r}}_{E E}$. Instead, it can be stabilized by the UAV attitude controller at an arbitrary orientation $\psi_{0}$, which is free but has to be known. Furthermore, to reduce the influence of motor inertia and friction, we again add the integral of the position error $\tilde{\boldsymbol{r}}=\boldsymbol{r}_{C M, d}-\boldsymbol{r}_{C M}$ to the system's state vector and design three independent linear controllers using pole placement [14]

$$
\begin{aligned}
& \ddot{r}_{E E, x, d}=-\boldsymbol{K}_{2}\left(\begin{array}{lllll}
\dot{r}_{x} & r_{x}-r_{x, 0} & \omega_{y} & \theta & \left.\int \tilde{r}_{x} \mathrm{~d} t\right)^{T},
\end{array}\right. \\
& \ddot{r}_{E E, y, d}=-\boldsymbol{K}_{3}\left(\begin{array}{lllll}
\dot{r}_{y} & r_{y}-r_{y, 0} & \omega_{x} & \varphi & \left.\int \tilde{r}_{y} \mathrm{~d} t\right)^{T},
\end{array}\right. \\
& \ddot{r}_{E E, z, d}=-\boldsymbol{K}_{4}\left(\begin{array}{lll}
\dot{r}_{z} & r_{z}-r_{z, 0} & \int \tilde{r}_{z} \mathrm{~d} t
\end{array}\right)^{T},
\end{aligned}
$$

instead of the pure translational controller (18). Therein, $\left(\begin{array}{lll}r_{x, 0} & r_{y, 0} & r_{z, 0}\end{array}\right)^{T}$ is the UAV's initial center of mass position. Inserting (26), (27), and (28) in (21) and using (19) for stabilizing the end-effector orientation at $\boldsymbol{R}_{d}=\boldsymbol{I}$ yields the feed-forward torque control law for the robot manipulator.

\section{Decoupling Position and Orientation Control of a UAV}

When we assume that it is possible to send orientation commands to the UAV, we gain a torque input $\Delta \boldsymbol{\tau}_{\text {att }}$ to the linear system dynamics, as depicted in Fig. 3,

$$
\begin{aligned}
& \left(\begin{array}{c}
\ddot{r}_{x} \\
\dot{r}_{x} \\
\dot{\omega}_{y} \\
\dot{\theta}
\end{array}\right)_{U A V}=\overline{\boldsymbol{A}}_{x}\left(\begin{array}{c}
\dot{r}_{x} \\
r_{x} \\
\omega_{y} \\
\theta
\end{array}\right)_{U A V}+\underbrace{\left[\begin{array}{cc}
1 & 0 \\
0 & 0 \\
-\frac{m l}{J_{y y}} & \frac{1}{J_{y y}} \\
0 & 0
\end{array}\right]}_{\overline{\boldsymbol{B}}_{x}} \underbrace{\left(\begin{array}{c}
\ddot{r}_{E E, x} \\
\Delta \tau_{a t t, y}
\end{array}\right)}_{\boldsymbol{u}_{x}}, \\
& \left(\begin{array}{c}
\ddot{r}_{y} \\
\dot{r}_{y} \\
\dot{\omega}_{x} \\
\dot{\varphi}
\end{array}\right)_{U A V}=\overline{\boldsymbol{A}}_{y}\left(\begin{array}{c}
\dot{r}_{y} \\
r_{y} \\
\omega_{x} \\
\varphi
\end{array}\right)_{U A V}+\underbrace{\left[\begin{array}{cc}
1 & 0 \\
0 & 0 \\
\frac{m l}{J_{x x}} & \frac{1}{J_{x x}} \\
0 & 0
\end{array}\right]}_{\overline{\boldsymbol{B}}_{y}} \underbrace{\left(\begin{array}{c}
\ddot{r}_{E E, y} \\
\Delta \tau_{a t t, x}
\end{array}\right)}_{\boldsymbol{u}_{y}},
\end{aligned}
$$




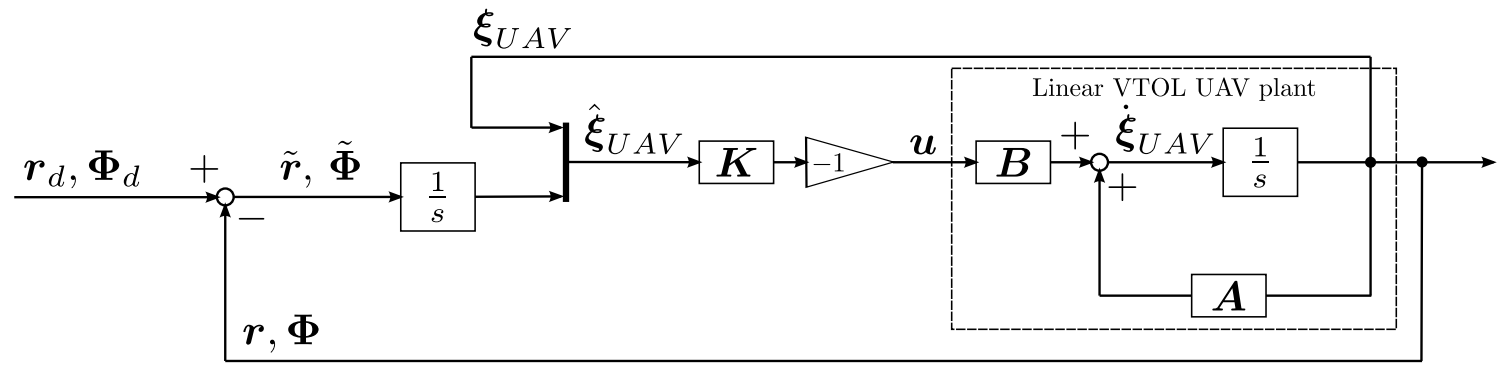

Fig. 4. General scheme of the linear state-space controller. The input $\boldsymbol{u}$ to the linear VTOL UAV model is the acceleration of the robot manipulator's end-effector. $\boldsymbol{\xi}_{U A V}$ denotes the vector of the VTOL UAV states and $\hat{\boldsymbol{\xi}}_{U A V}$ denotes the extended state vector including the integrals of the position error $\tilde{\boldsymbol{r}}$ and the orientation error $\tilde{\boldsymbol{\Phi}}$. The control gain $\boldsymbol{K}$ represents the controllers (18), (26) - (28), and (34) - (35), which use different manifestations of $\hat{\boldsymbol{\xi}}_{U A V}$.

through which $\psi$ is now controllable as well:

$$
\begin{aligned}
& \left(\begin{array}{c}
\ddot{r}_{z} \\
\dot{r}_{z}
\end{array}\right)_{U A V}=\left[\begin{array}{ll}
0 & 0 \\
1 & 0
\end{array}\right]\left(\begin{array}{c}
\dot{r}_{z} \\
r_{z}
\end{array}\right)_{U A V}+\left(\begin{array}{l}
1 \\
0
\end{array}\right) \ddot{r}_{z}, \\
& \left(\begin{array}{c}
\dot{\omega}_{z} \\
\dot{\psi}
\end{array}\right)_{U A V}=\boldsymbol{A}_{z}\left(\begin{array}{c}
\omega_{z} \\
\psi
\end{array}\right)_{U A V}+\left(\begin{array}{c}
\frac{1}{J_{z z}} \\
0
\end{array}\right) \Delta \tau_{a t t, z} .
\end{aligned}
$$

Again, we add the integral of the position error $\tilde{r}$ and additionally the integral of the orientation error $\tilde{\boldsymbol{\Phi}}=\left(\begin{array}{lll}\tilde{\varphi} & \tilde{\theta} & \tilde{\psi}\end{array}\right)^{T}=\left(\begin{array}{lll}\varphi_{d}-\varphi & \theta_{d}-\theta & \psi_{d}-\psi\end{array}\right)^{T}$ to the system states. The matrices $\overline{\boldsymbol{A}}_{x}, \overline{\boldsymbol{A}}_{y}, \overline{\boldsymbol{B}}_{x}$, and $\overline{\boldsymbol{B}}_{y}$ are adapted accordingly and become

$$
\overline{\boldsymbol{A}}_{x / y}^{*}=\left[\begin{array}{ccccc} 
& \overline{\boldsymbol{A}}_{x / y} & & \mathbf{0} \\
0 & -1 & 0 & 0 & \mathbf{0} \\
0 & 0 & 0 & -1 &
\end{array}\right], \overline{\boldsymbol{B}}_{x / y}^{*}=\left[\begin{array}{c}
\overline{\boldsymbol{B}}_{x / y} \\
\mathbf{0}
\end{array}\right] .
$$

We seek to find decoupling control laws

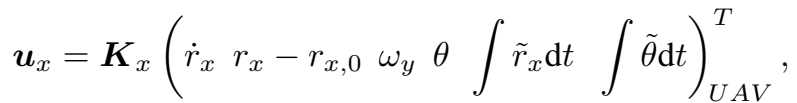

$$
\begin{aligned}
& \boldsymbol{u}_{y}=\boldsymbol{K}_{y}\left(\dot{r}_{y} r_{y}-r_{y, 0} \omega_{x} \varphi \int \tilde{r}_{y} \mathrm{~d} t \int \tilde{\varphi} \mathrm{d} t\right)_{U A V}^{T}
\end{aligned}
$$

for (29) and (30) and use a formula by Roppenecker [17] to compute the gains $\boldsymbol{K}_{x}$ and $\boldsymbol{K}_{y}$

$$
\boldsymbol{K}_{x / y}=\left[\boldsymbol{p}_{1} \ldots \boldsymbol{p}_{6}\right]_{x / y}\left[\boldsymbol{w}_{1} \ldots \boldsymbol{w}_{6}\right]_{x / y}^{-1} .
$$

Therein, $\boldsymbol{p}_{i, x / y}$ with $i \in\{1,2,3,4,5,6\}$ are the so-called parameter vectors defined as $\boldsymbol{p}_{i, x / y}=\boldsymbol{K}_{x / y} \boldsymbol{w}_{i, x / y}$, with $\boldsymbol{w}_{i, x / y}$ being the eigenvectors associated with the eigenvalues $\lambda_{i, x / y}$ of the closed-loop system (29) with (34), or (30) with (35), respectively. From the definition of the eigenvectors, the following linear system of equations is obtained

$$
\underbrace{\left[\begin{array}{cc}
\lambda_{i} \boldsymbol{I}-\overline{\boldsymbol{A}}^{*} & \overline{\boldsymbol{B}}^{*} \\
\boldsymbol{e}_{j(i)}^{T}
\end{array}\right]_{x / y}}_{\boldsymbol{D}_{i, x / y}}\left(\begin{array}{l}
\boldsymbol{w}_{i} \\
\boldsymbol{p}_{i}
\end{array}\right)_{x / y}=\mathbf{0 .}
$$

The unit row vectors $\boldsymbol{e}_{j(i)}^{T}$, whose elements are all zero except for the $j$ th element which is one, are added in (37) in order to make the system of equations solvable and allow to define additional requirements for the solution of (37). We want to suppress certain states in the eigenmodes $\exp \left(\lambda_{i}\left(t-t_{0}\right)\right) \boldsymbol{w}_{i, x / y}$ of the closed-loop systems and formulate the requirements as follows: The eigenvalue $\lambda_{i, x / y}$ should not influence the $j$ th state with $i \in\{1,2,3,4,5,6\}$ and $j \in\{1,2,3,4,4,2\}$. The solution of (37) is obtained from $\left(\begin{array}{ll}\boldsymbol{w}_{i} & \boldsymbol{p}_{i}\end{array}\right)_{x / y}^{T}=\operatorname{kernel}\left(\boldsymbol{D}_{i, x / y}\right)$ analytically using the six $7 \times 8$ matrices $\boldsymbol{D}_{i, x / y}$ yielding

$$
\boldsymbol{K}_{x}=\left[\begin{array}{cc}
-a & -m \cdot l \cdot a \\
b & m \cdot l \cdot b \\
0 & -k_{\omega_{y}}-J_{y y} \cdot d \\
g & -k_{\theta}+2 \cdot m \cdot g \cdot l+J_{y y} \cdot e \\
c & m \cdot l \cdot c \\
0 & J_{y y} \cdot f
\end{array}\right]^{T}
$$

with

$$
\begin{array}{lll}
a=\lambda_{3}+\lambda_{4}+\lambda_{5}, & b=\lambda_{3} \lambda_{4}+\lambda_{3} \lambda_{5}+\lambda_{4} \lambda_{5}, \\
c=\lambda_{3} \lambda_{4} \lambda_{5}, & d=\lambda_{1}+\lambda_{2}+\lambda_{6}, \\
e=\lambda_{1} \lambda_{2}+\lambda_{1} \lambda_{6}+\lambda_{2} \lambda_{6}, & f=\lambda_{1} \lambda_{2} \lambda_{6} .
\end{array}
$$

From the closed-loop equation for the $x$-direction

$$
\overline{\boldsymbol{A}}_{x}^{*}-\overline{\boldsymbol{B}}_{x}^{*} \boldsymbol{K}_{x}=\left[\begin{array}{cccccc}
a & -b & 0 & 0 & -c & 0 \\
1 & 0 & 0 & 0 & 0 & 0 \\
0 & 0 & d & -e & 0 & -f \\
0 & 0 & 1 & 0 & 0 & 0 \\
0 & -1 & 0 & 0 & 0 & 0 \\
0 & 0 & 0 & -1 & 0 & 0
\end{array}\right],
$$

it can be seen that the translational and the rotational states are indeed decoupled if the controller (38) is used.

The controller gain $\boldsymbol{K}_{y}$ follows analogously, but is omitted here for the sake of brevity. The controllers for the subsystems (31) and (32) with gains $\boldsymbol{K}_{z}$ and $\boldsymbol{K}_{\psi}$ are again obtained using pole placement and with the integral of the position and the orientation error $\tilde{r}_{z}$ and $\tilde{\psi}$, respectively, as additional states.

\section{EXPERIMENTAL EVALUATION}

Our landing system demonstrator is shown in Fig. 5 and a short video of it is provided online [18]. It is composed of a torque-controlled DLR/KUKA light-weight robot (LWR) with seven degrees-of-freedom [15]. At the end-effector, a camera system for visually tracking the UAV, an electromagnet for attaching and releasing the aerial vehicle, and a force-torque-sensor for measuring the interaction forces are mounted. The off-the-shelf quadrotor AR.Drone 2.0 [12], which is used for lab experiments, is equipped with a custom-built universal hinge that decouples UAV and endeffector orientation such that only translational forces are applied by the robot (see Fig. 5). The quadrotor's model 
TABLE I

MODEL PARAMETERS OF QUADROTOR AR.DRONE 2.0

\begin{tabular}{|c|c|c|}
\hline Mass $m[\mathrm{~kg}]$ & Inertia $\boldsymbol{J}\left[\mathrm{kg} \mathrm{m}^{2}\right]$ & Hinge length $l[\mathrm{~m}]$ \\
\hline 0.480 & 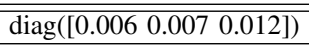 & 0.06 \\
\hline
\end{tabular}

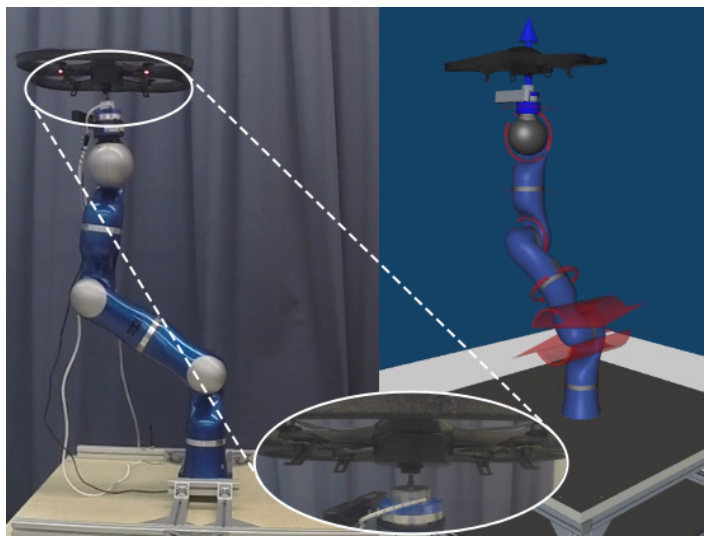

Fig. 5. Robotic VTOL UAV landing system in the DLR Flying Robots lab (left) and a screenshot from the simulation environment (right). The enlarged detail shows the universal hinge and the electromagnet which connect the quadrotor to the end-effector of the serial robotic manipulator.

parameters can be found in Table I and its attitude controller gains are identified to be $\left(k_{\varphi}, k_{\theta}, k_{\psi}, k_{\omega_{x}}, k_{\omega_{y}}, k_{\omega_{z}}\right)=$ $(0.59,0.66,0.03,0.04,0.05,0.03)$. The gravitional acceleration is assumed to be $g=9.81 \mathrm{~m} / \mathrm{s}^{2}$. All controllers are implemented using MATLAB/Simulink and the communication with the quadrotor is established using standard $2.4 \mathrm{GHz}$ wireless LAN.

The gain for the model-free controller $\boldsymbol{K}_{1}$ is obtained by placing the poles at $\lambda_{i, 1} \in\{-3,-4,-5\}$, which results in a subjectively fast motion of the LWR. The poles for the coupled controller are chosen as $\lambda_{i, 2 / 3} \in\{-5,-5,-5,-5,-5\}$ and $\lambda_{i, 4} \in\{-3,-4,-5\}$ yielding $\boldsymbol{K}_{2 / 3}$ and $\boldsymbol{K}_{4}$, respectively. We choose $\lambda_{i, 2 / 3}$ faster than the open-loop poles to see if the orientation control is nevertheless better compared to the model-free controller. The poles for the decoupling controller are chosen with slightly different orientation eigenvalues $\lambda_{1, x / y}, \lambda_{2, x / y}$, and $\lambda_{6, x / y}$ compared to the openloop, as $\lambda_{i, x / y} \in\{-3.5+9 \mathrm{i},-3.5-9 \mathrm{i},-3,-4,-5,-5\}$ and $\lambda_{i, z / \psi} \in\{-3,-3,-3\}$ yielding $\boldsymbol{K}_{x / y}$ and $\boldsymbol{K}_{z / \psi}$ respectively, to examine the influence of the decoupling in the closed-loop behaviour. The end-effector of the robot is controlled such that it always points upwards, as shown in Fig. 5, using the orientation controller (19) with gains $\left(k_{E E}, k_{\omega_{E E}}\right)=(12.0,7.0)$. In order to resolve the joint redundancy of the LWR, the desired elbow configuration points downwards and is realized with an elbow field [19] which produces $\boldsymbol{\tau}_{n s p}$.

All three controllers are tested with the same sequence of four position set-points as shown in the top row of Fig. 8 - 10 for the controllers III-A - III-C and exemplarily in Fig. 7 for the decoupling controller from Section III-C. No additional interpolator is used between the desired set-points. Note that for the model-free controller from Section III-A the set-point for the end-effector ${ }^{i} \boldsymbol{r}_{E E, d}$ is commanded, while for the coupled (Section III-B) and the decoupling controller (Section III-C) the desired position of the UAV's center of

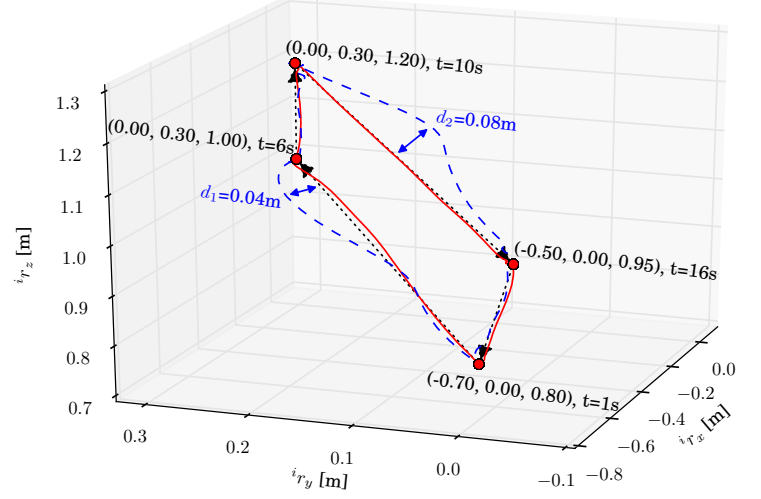

Fig. 6. Path of ${ }^{i} \boldsymbol{r}_{C M}$ in the experiments with the coupled (-) and the decoupling controller (- -). The arrows indicate the direction of the motion. For the decoupling controller two maximum deviations $d_{1}$ and $d_{2}$ from the direct path between two waypoints are depicted.

mass ${ }^{i} \boldsymbol{r}_{C M, d}$ is commanded. Every set-point is sent as soon as the previous waypoint is reached within $1 \mathrm{~cm}$ accuracy. Hence, the duration of the trajectory segments for the three different controllers varies, as can be seen in the top row of Fig. $8-10$. With the model-free controller it takes about $19 \mathrm{~s}$ to execution the complete trajectory sequence, the coupled controller needs approximately $17 \mathrm{~s}$ and the decoupling controller is slowest with about $20 \mathrm{~s}$. The mean squared error (MSE) of the deviation from the four position set-points is very similar for the model-free (MSE: 0.028), the coupled (MSE: 0.029), and the decoupling controller (MSE: 0.029). However, the path of the UAV's center of mass in Fig. 6 reveals a deviation from the direct connection between two waypoints of up to $8 \mathrm{~cm}$ for the decoupling controller.

The desired orientation of the quadrotor, written in Euler angles, is $\left(\begin{array}{lll}\varphi & \theta & \psi\end{array}\right)^{T}=\left(\begin{array}{lll}0 & 0 & 0\end{array}\right)^{T}$ and its trajectory is shown in the second row of Fig. 8 - 10. The MSE of the deviation from the desired orientation of the Euler angles $\varphi, \theta, \psi$, depicted in Fig. 8 - 10, shows that the precision of the orientation control using the decoupling controller is superior compared to the other two controllers (MSE: 1.81). The model-free controller shows the worst performance in orientation control (MSE: 3.77). The third row of Fig. 8 - 10 depicts the corresponding angular velocities of the quadrotor measured by its onboard IMU. The desired and measured joint torques of the robot manipulator are depicted in the last row of Fig. 8 - 10 and show equal tracking performance in all three experiments of the low-level torque controller of the LWR. The additional torque commands for the quadrotor's attitude controller $\Delta \boldsymbol{\tau}_{\text {att }}$ are only shown in the fourth row of Fig. 10 since $\Delta \tau_{a t t}$ is only used with this controller (see also Fig. 3).

Our experimental results indicate that the coupled and the decoupling controller designed in this work both increase the performance in position and attitude control compared to a model-free controller. The coupled controller can fulfill position and orientation control simultaneously while the weighting between both is defined by the eigenvalues of the closed-loop system. With the selected eigenvalues, the 


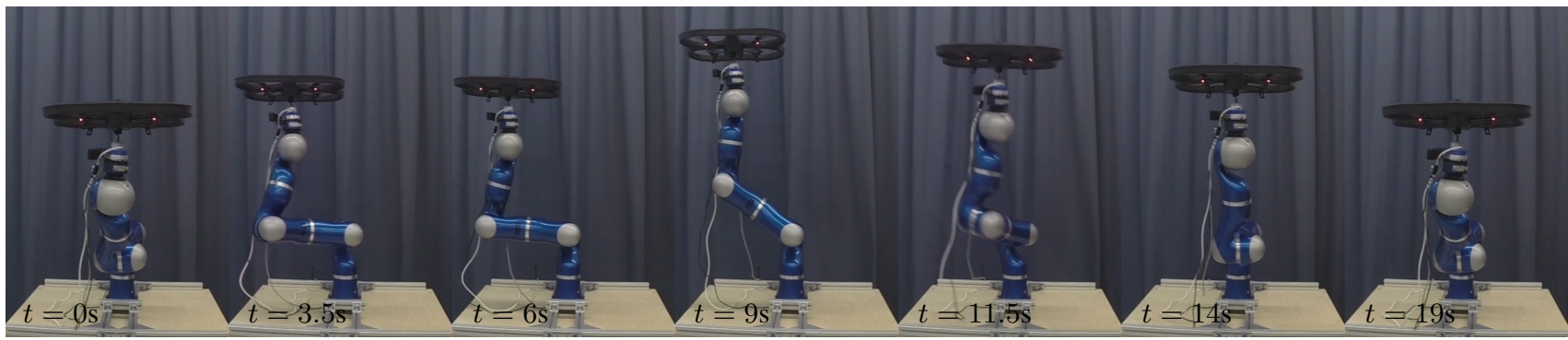

Fig. 7. Picture sequence from the experiment with the controller from Section III-C, the indicated time instants correspond to the plot in Fig. 10. A short video by the authors of this paper showing the VTOL UAV landing system demonstrator and some of the conducted experiments is provided online [18].
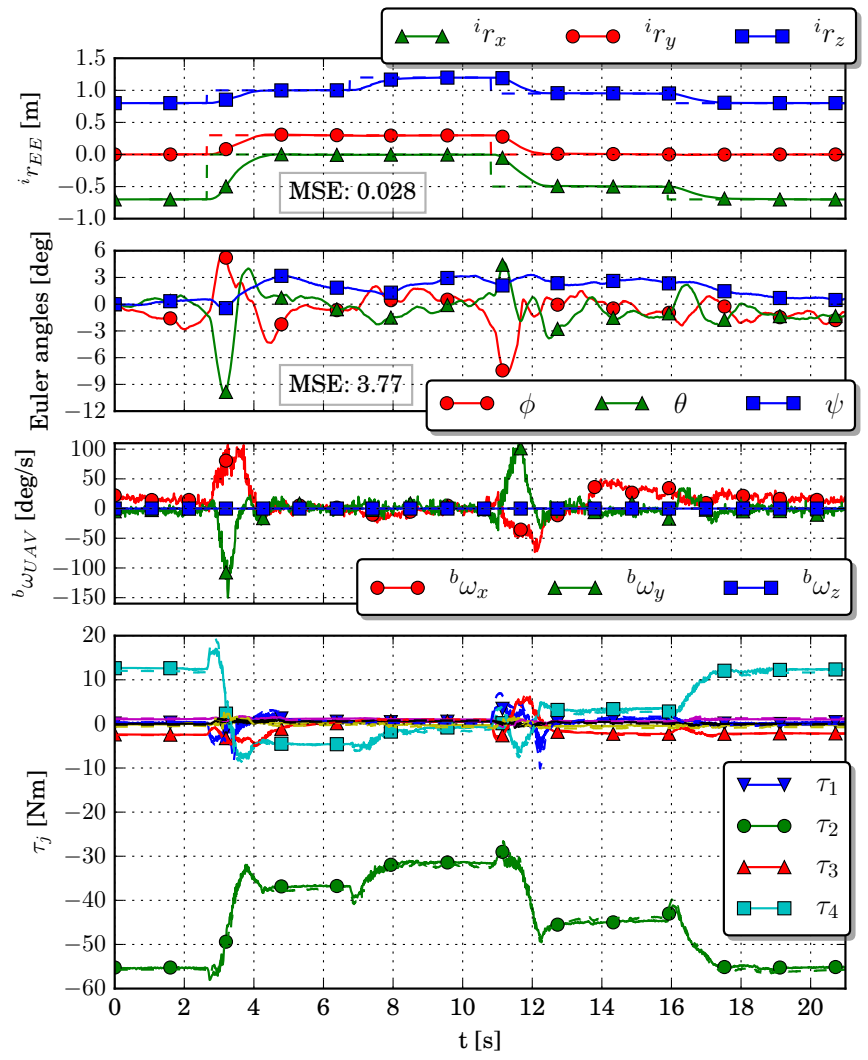

Fig. 8. Experimental results using the model-free controller from Section III-A. The top row shows the trajectory of the robot's end-effector. The last row shows the joint torques of the light-weight robot. Only the first four torques are labeled since the other three are close to zero.

execution time of the test sequence is reduced by $2 \mathrm{~s}$ while the accuracy in orientation control is increased. The decoupling controller mitigates the effect of a change in the UAV's orientation on its center of mass position in the inertial frame and in addition generates correction torques for the UAV's attitude. This leads to deviations from the direct path between two waypoints, but to best performance in attitude control compared to the other two controllers. The reason for the deviation in position is, that desired set-points are commanded instead of a continuous trajectory between the four points in cartesian space. However, the attitude torque of the UAV is not controlled very accurately throughout the experiments, which is why the disturbance is not compensated completely. In practice, the difference in performance of the coupled and the
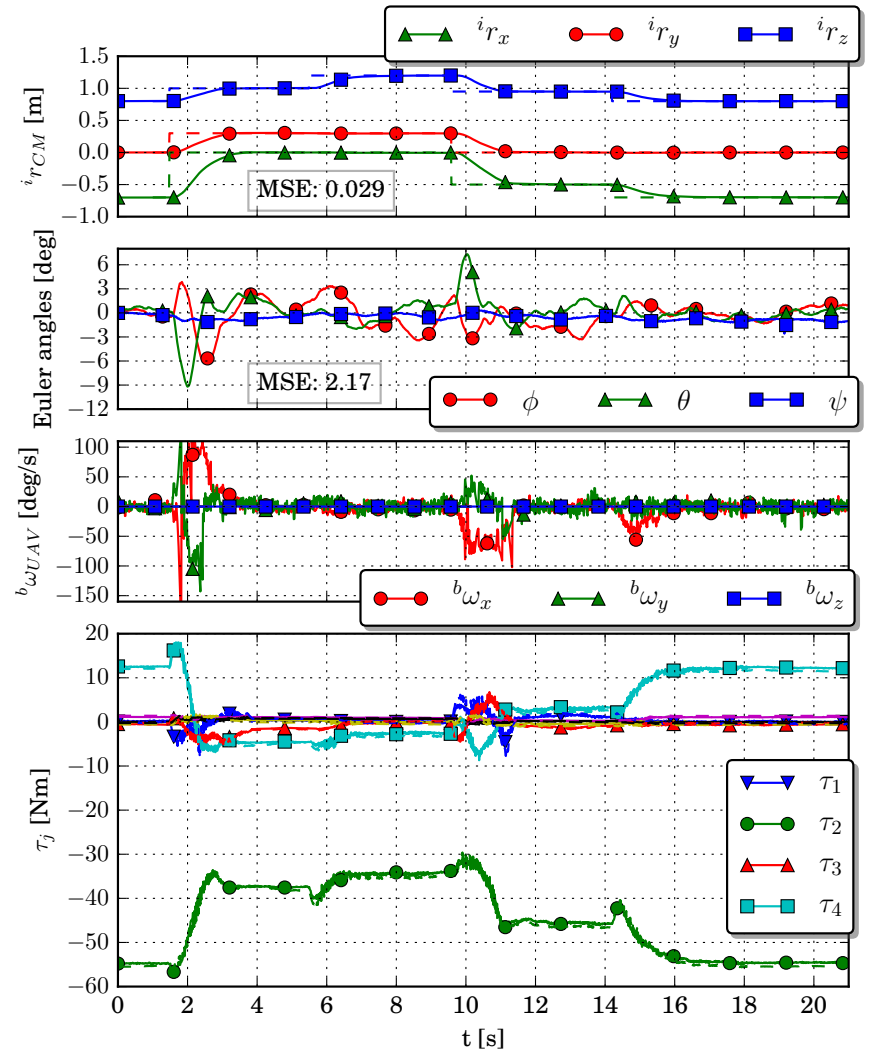

Fig. 9. Experimental results using the coupled controller from Section III-B. The top row shows the trajectory of the UAV's center of mass.

decoupling controller will highly depend on the acceleration control of the robot as well as on the torque control of the UAV. The coupled controller only uses state feed-back from the UAV, while for the decoupling controller it is important that the computed torque $\Delta \boldsymbol{\tau}_{\text {att }}$ is really produced by the UAV. Therefore, the coupled controller is beneficial for applications where no accurate UAV torque controller is available.

\section{CONCLUSION AND OUTLOOK}

In summary, we consider a novel landing system for UAV rotorcrafts that uses a robot manipulator to capture and land the UAV. We present a rigid-body model of an attitude-controlled VTOL UAV fixed to a manipulator via a universal hinge and derive a stability criterion for linear PD attitude controllers in the static case, i.e. when the robot is not moving. The 

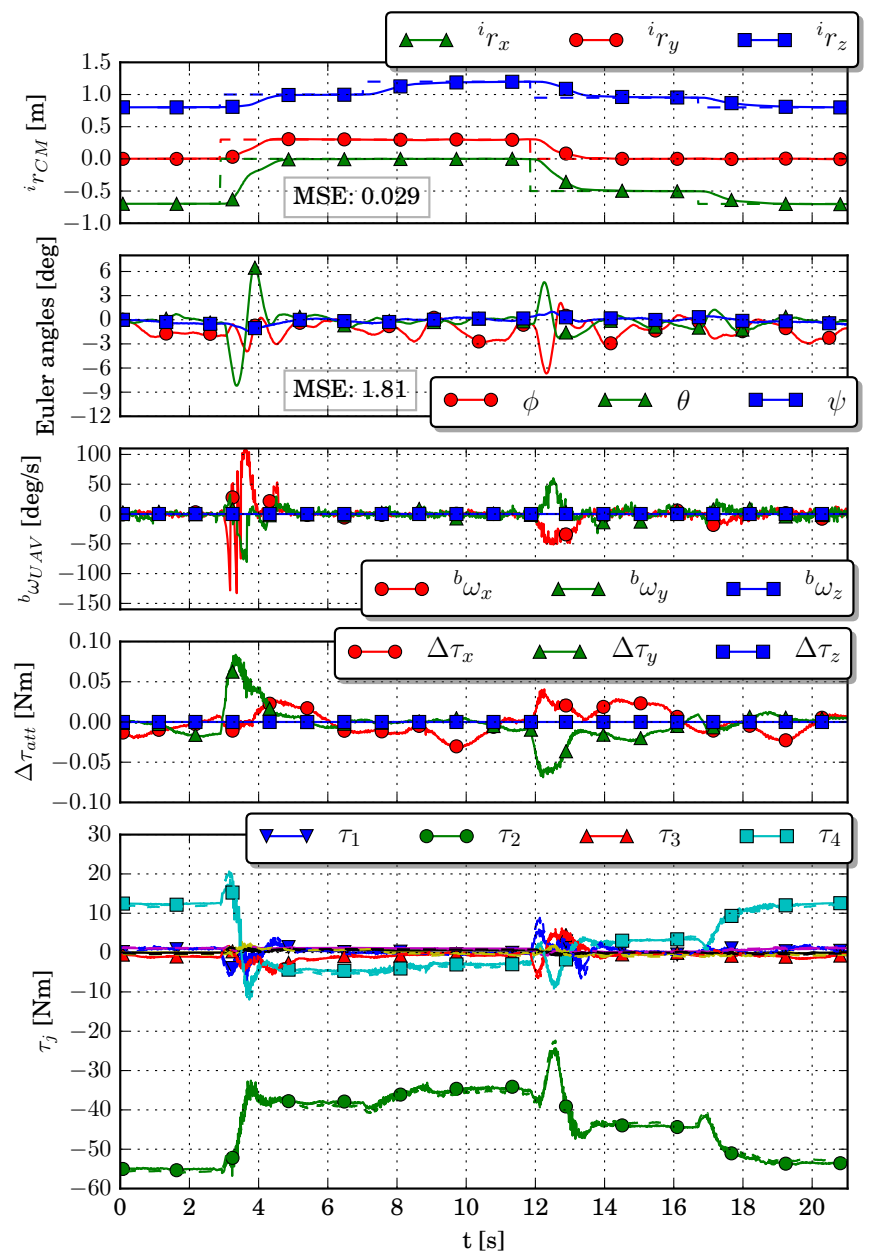

Fig. 10. Experimental results using the decoupling controller from Section III-C. The fourth row shows the attitude torques which are sent to the aerial vehicle.

linearized model of the UAV is used to design a coupled and a decoupling state-space controller for the landing system assuming that the acceleration of the pivot point of the hinge can be controlled directly. It is assumed that the gains of the PD attitude controller are known and that the thrust of the $\mathrm{UAV}$ is constant with $f_{T}=m g$. We evaluate the performance of the controllers in experiments with a KUKA/DLR lightweight robot and an AR.Drone 2.0 quadrotor. Compared to a controller which does not use a model of the UAV, the precision of the attitude control of the UAV is increased with both model-based controllers. The coupled controller uses state-feedback from the UAV and has the advantage that no commands have to be sent to the aerial vehicle. The decoupling controller is realized by sending desired torques to the UAV and compensates for the disturbance imposed by a change of the UAV's attitude.

The results provided in this paper show, that the presented control strategy is applicable to the novel robotic VTOL UAV landing system. We plan to use a more elaborated model of the UAV connected to the manipulator in order to increase the performance of the controllers even further. Currently, we are working on a floating base model of the manipulator in order to account for platform movements, e.g. ship motions. In the next step, we are going to incorporate disturbance observation and base motion compensation as well as actuator limits of the aerial vehicle in the control approach.

\section{ACKNOWLEDGMENTS}

The authors would like to thank Craig Mathews, Shane Patch, James Forrest, and Ben Chartier of Prism Defence, Australia, for providing valuable information regarding ship helicopter integration and landing using a serial robotic manipulator.

\section{REFERENCES}

[1] T. Tomic, M. Maier, and S. Haddadin, "Learning quadrotor maneuvers from optimal control and generalizing in real-time," in Proc. IEEE Int. Conf. on Robotics and Automation (ICRA), May 2014.

[2] J. Forrest, I. Owen, G. Padfield, and S. Hodge, "Ship-helicopter operating limits prediction using piloted flight simulation and timeaccurate airwakes," J. of Aircraft, vol. 49, no. 4, pp. 1020-1031, July 2012. [Online]. Available: http://dx.doi.org/10.2514/1.C031525

[3] J. Sanchez-Lopez, S. Saripalli, P. Campoy, J. Pestana, and C. Fu, "Toward visual autonomous ship board landing of a VTOL UAV," in Proc. Int. Conf. on Unmanned Aircraft Systems (ICUAS), May 2013, pp. 779-788.

[4] P. Vlantis, P. Marantos, C. P. Bechlioulis, and K. J. Kyriakopoulos, "Quadrotor landing on an inclined platform of a moving ground vehicle," in Proc. IEEE Int. Conf. on Robotics and Automation (ICRA), May 2015, pp. 2202-2207.

[5] J. Hervas, M. Reyhanoglu, and H. Tang, "Automatic landing control of unmanned aerial vehicles on moving platforms," in Proc. IEEE Int. Symp. on Industrial Electronics (ISIE), June 2014, pp. 69-74.

[6] L. Sandino, M. Bejar, and A. Ollero, "On the applicability of linear control techniques for autonomous landing of helicopters on the deck of a ship," in Proc. IEEE Int. Conf. on Mechatronics (ICM), April 2011, pp. $363-368$.

[7] A. Isidori, L. Marconi, and A. Serrani, "Robust nonlinear motion control of a helicopter," IEEE Trans. on Automatic Control, vol. 48, no. 3, pp. 413-426, March 2003. [Online]. Available: http://dx.doi.org/10.1109/TAC.2003.809147

[8] A. Campos, J. Quintero, R. Saltaren, M. Ferre, and R. Aracil, "An active helideck testbed for floating structures based on a stewart-gough platform," in Proc. IEEE/RSJ Int. Conf. on Intelligent Robots and Systems (IROS), Sept 2008, pp. 3705-3710.

[9] S.-R. Oh, K. Pathak, S. Agrawal, H. Pota, and M. Garratt, "Approaches for a tether-guided landing of an autonomous helicopter," IEEE Trans. on Robotics, vol. 22, no. 3, pp. 536-544, June 2006.

[10] L. Sandino, D. Santamaria, M. Bejar, A. Viguria, K. Kondak, and A. Ollero, "Tether-guided landing of unmanned helicopters without GPS sensors," in Proc. IEEE Int. Conf. on Robotics and Automation (ICRA), May 2014, pp. 3096-3101.

[11] S. A. Conyers, N. I. Vitzilaios, M. J. Rutherford, and K. P. Valavanis, "A mobile self-leveling landing platform for vtol uavs," in Proc. IEEE Int. Conf. on Robotics and Automation (ICRA), May 2015, pp. 815-822.

[12] M. Maier and K. Kondak, "Landing of vtol uavs using a stationary robot manipulator: A new approach for coordinated control," in IEEE Conf. on Decision and Control (CDC), December 2015, accepted.

[13] P. Pounds and A. Dollar, "Stability of helicopters in compliant contact under pd-pid control," IEEE Trans. on Robotics, vol. 30, no. 6, pp. 1472-1486, Dec 2014.

[14] F. W. Fairman, Linear Control Theory: The State Space Approach. John Wiley \& Sons, 1998.

[15] C. Ott, Cartesian Impedance Control of Redundant and Flexible-Joint Robots. Springer Science \& Business Media, Aug 2008.

[16] M. J. Sidi, Spacecraft Dynamics and Control: A Practical Engineering Approach, 1997.

[17] G. Roppenecker, "On parametric state feedback design," International Journal of Control, vol. 43, no. 3, pp. 793-804, 1986.

[18] M. Maier, A. Oeschger, and K. Kondak, "Robot-assisted landing of vtol uavs," https://youtu.be/r6_uVIhORAg, German Aerospace Center (DLR), Institute of Robotics and Mechatronics, Münchener Straße 20, 82234 Weßling, Germany, September 2015, [Online; accessed Nov. 12, 2015].

[19] F. Huber, K. Kondak, K. Krieger, D. Sommer, M. Schwarzbach, M. Laiacker, I. Kossyk, S. Parusel, S. Haddadin, and A. Albu-Schaffer, "First analysis and experiments in aerial manipulation using fully actuated redundant robot arm," in Proc. IEEE/RSJ Int. Conf. on Intelligent Robots and Systems (IROS), Nov 2013, pp. 3452-3457. 